\title{
Circulating Tumour DNA in Melanoma—Clinic Ready?
}

\author{
Ann Tivey ${ }^{1,2} \cdot$ Fiona Britton ${ }^{1} \cdot$ Julie-Ann Scott ${ }^{1} \cdot$ Dominic Rothwell ${ }^{2,3} \cdot$ Paul Lorigan $^{1,2} \cdot$ Rebecca Lee $^{1,2,4}$ (D)
}

Accepted: 4 October 2021 / Published online: 8 February 2022

(c) The Author(s) 2022

\begin{abstract}
Purpose of Review Liquid biopsies, including circulating tumour DNA (ctDNA), can inform a variety of clinical questions. This review examines the potential role of ctDNA as a clinical tool to inform clinical decision-making from early to late stage cutaneous melanoma.

Recent Findings In pre-clinical studies, ctDNA has been shown to detect minimal residual disease and molecular relapse; predict and monitor response to therapy; and identify key resistance mechanisms. Here, we examine the potential utility of ctDNA and discuss its limitations for use in patients with melanoma. We present novel clinical trials, which are testing its value as a tool to augment clinical decision-making. Finally, we discuss the steps that are needed to ensure that ctDNA is used optimally in order to improve outcomes for patients with melanoma.

Summary Preclinical studies have shown that ctDNA has huge potential to provide real-time information about disease status in patients with melanoma. It is now time to test it rigorously within clinical trials to assess how it can be optimally used to benefit patients in the clinic.
\end{abstract}

Keywords ctDNA $\cdot$ Liquid biopsies $\cdot$ Melanoma $\cdot$ Immunotherapy $\cdot$ Targeted therapy $\cdot$ Biomarkers

\section{Introduction}

There have been huge therapeutic advances in the treatment of both early and advanced melanoma over the past two decades, with targeted therapies and immune checkpoint inhibitors (ICI) significantly improving progression-free and overall survival. In parallel, with the development of effective treatment options, the concept of 'precision' or 'personalised' medicine has gained traction, whereby timing and choice of treatment are tailored to an individual patient and

This article is part of the Topical Collection on Melanoma

Rebecca Lee

Rebecca.lee-3@manchester.ac.uk

1 The Christie NHS Foundation Trust, Wilmslow Road, Manchester M20 4BX, UK

2 Division of Cancer Sciences, The University of Manchester, Oxford Road, Manchester M13 9PL, UK

3 Nucleic Acids Biomarker Team, Cancer Research UK Manchester Institute, Cancer Biomarker Centre, The University of Manchester, Alderley Park SK10 4TG, UK

4 Department of Medical Oncology, The Christie NHS Foundation Trust, Wilmslow Road, Manchester M20 4BX, UK their tumour. Blood-based biomarkers such as circulating tumour DNA (ctDNA), circulating tumour cells (CTCs) and other 'liquid biopsies' have the potential to play a key role in enabling precision strategies to be delivered. This review will focus on the use of ctDNA for clinical applications of liquid biopsies; however, assays including CTC enumeration and analysis can provide additional information about the cancer such as gene expression/protein changes, which will also be important for the clinic.

Cell-free DNA (cfDNA) is DNA that freely circulates in the bloodstream and consists of fragments that are on average 140 to 170 base pairs (bp) long [1]. CfDNA is found in the blood stream of healthy subjects at an average concentration of $2 \mathrm{ng} / \mathrm{ml}$ (range $1-6 \mathrm{ng} / \mathrm{ml}$ ) and in patients with lung cancer an average of $8 \mathrm{ng} / \mathrm{ml}$ (range $1-41 \mathrm{ng} / \mathrm{ml}$ ) [2]. The mechanisms of cfDNA release are not completely understood; however, it is thought it is produced by cell necrosis, apoptosis and secretion from macrophages that have phagocytosed cells $[1,3]$. Circulating tumour DNA originates from cancer cells and contributes to total cfDNA in the blood, thereby increasing the concentration of cfDNA when cancer is present. Studies have shown that there is enrichment of cfDNA fragment sizes of between 90 and 150 base 
pairs in patients with cancer compared to healthy volunteer blood but also a lower enrichment at 250-300 base pairs [4].

Mandel and Métais first identified circulating nucleic acids in the blood stream in 1948; however, it was not until 1994 that their potential utility as biomarkers for cancer detection and monitoring was realised [1]. At that time, presence of mutated KRAS ctDNA sequences were identified in blood of patients with pancreatic cancer with KRAS mutant tumours [5••]. Many studies since then have examined the role of ctDNA as a 'liquid biopsy' which can predict response to treatment, monitor clinical response, identify timing of disease progression and elucidate mechanisms of resistance to therapy.

In this review, we will discuss the potential role of ctDNA as a clinical tool to inform clinical decision-making from early to late stage cutaneous melanoma. We reflect on the current evidence base for use of ctDNA in the clinic and its limitations. Finally, we will examine trials that are using ctDNA to inform real-time clinical decisions and propose how ctDNA could be developed to aid future treatment strategies (Fig. 1).

\section{Analysis of Circulating Tumour DNA}

There are a huge number of different methods to analyse ctDNA, from digital techniques analysing specific point mutations, to targeted panels (generally analysing up to 1000 genes), to broader approaches using whole exome sequencing (WES) and methylation-based assays. These have been extensively reviewed elsewhere and therefore will not be discussed in detail in this review $[1,6,7]$. However, it is important to note that determining the right assay to use for different clinical scenarios is critical when considering the application of ctDNA to the clinic. For example, in developing an assay for minimal residual disease detection (MRD), broad but low depth whole exome sequencing is unlikely to have sufficient sensitivity to identify micro-metastatic disease; however, this technique may be more useful in characterising novel resistance mechanisms to therapy. Each assay has advantages and limitations, which affect its utility for a specific scenario. Newer techniques such as MRD-EDGE using machine learning-based denoising and an expanded feature space including fragmentomics and allelic frequency of germline single nucleotide polymorphisms are however changing this paradigm. Thus, when designing trials

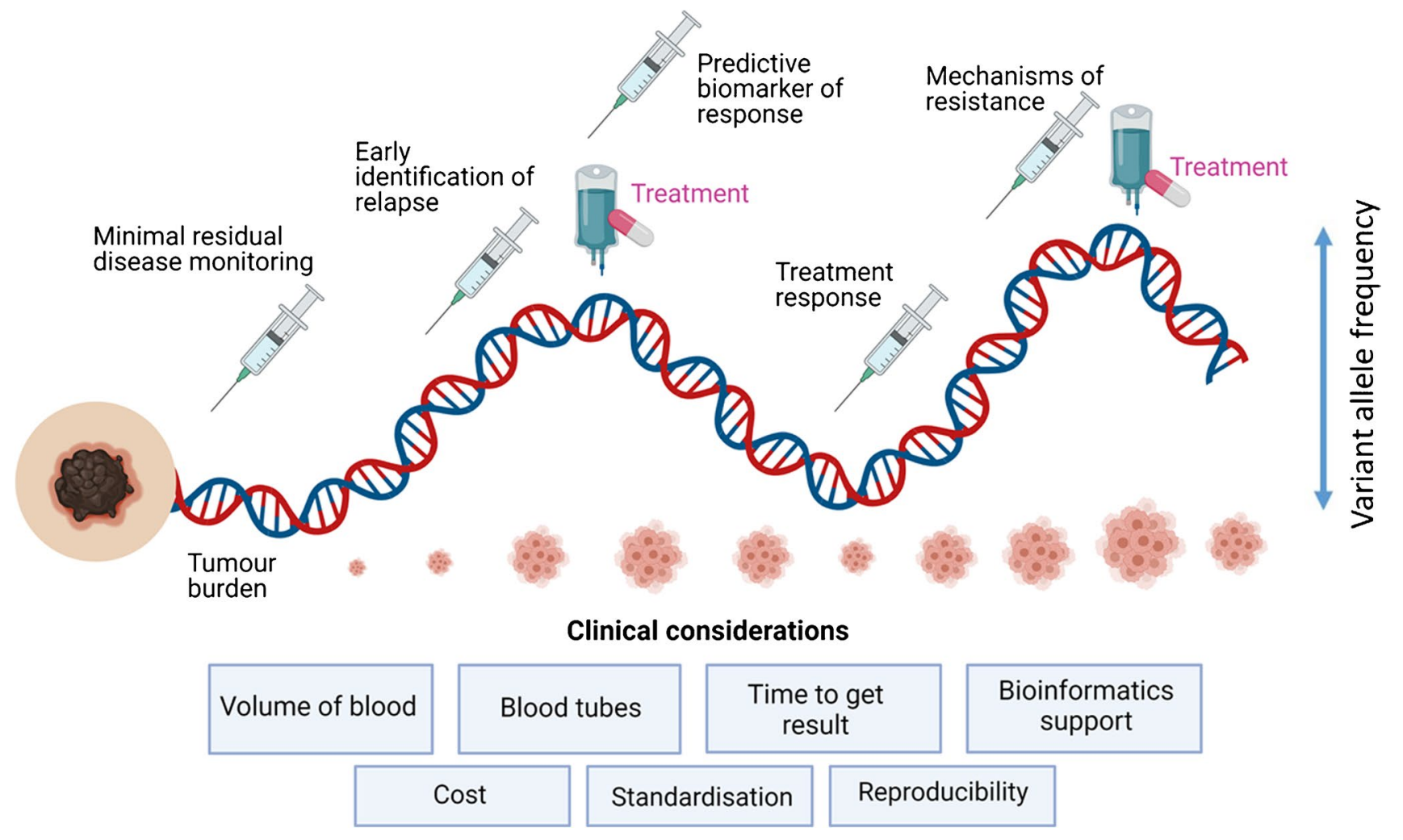

Fig. 1 Potential clinical utility of ctDNA throughout melanoma disease stages 
using ctDNA, clinicians should ask themselves what information is most important to obtain for the patient at that particular stage of disease in order to address the clinical question, then tailor the ctDNA assay accordingly. It will also be important, if ctDNA moves beyond the trial setting and into the clinic, that clinicians understand the performance of the test they are using in their patient population and appreciate its limitations if they are to make treatment decisions on the basis of its results. In addition, in some cancers, driver mutations (e.g. BRAF V600) are easier to identify than others where multi-regional sequencing might be required to identify truncal mutations. This impacts on the ability to select single-point mutations to assess tumour burden. Other clinical considerations include ease of sample collection and initial laboratory processing, requirement for bioinformatics support in interpreting the results and overall cost in performing the assays, especially if longitudinal monitoring is proposed.

\section{Identification of Minimal Residual Disease and Early Relapse Monitoring}

Adjuvant therapy with either targeted (dabrafenib plus trametinib $[\mathrm{D}+\mathrm{T}]$ ) or ICI (pembrolizumab or nivolumab) is now standard of care for patients with stage III melanoma based on data showing improved relapse-free survival (RFS) [8-11]. However, these treatments are not without side effects and ICI, in particular, is associated with long-term endocrine toxicities, which may impact future quality of life for patients [12]. As such, it is important that adjuvant therapy is offered to patients who will gain the greatest benefit, and lower-risk patients spared from possible life-threatening and lifelong toxicities. There are now data on the prognostic role of ctDNA in patients with melanoma who have undergone curative-intent surgical resection at pre-operative, postoperative and post-adjuvant time points.

Detection of ctDNA pre-operatively in patients with stage III melanoma is predictive of relapse independent of standard American Joint Committee on Cancer (AJCC) staging [13•, $14 \bullet \bullet$. In a study of 58 patients with stage III (A-D) melanoma who had pre-operative plasma samples, 21 (36\%) patients had detectable levels of ctDNA with 19 (90\%) of these patients relapsing during the 20-month follow-up period (relapse-free survival (RFS) HR 2.9; 95\% CI 1.5-5.6; $\mathrm{P}=0.002$ ). Of note, median time to relapse was 6.2 months for those with detectable ctDNA vs. 10.8 months in 18 (49\%) of patients who relapsed within the follow-up period, but did not have detectable ctDNA pre-operatively [14••]. In parallel, another group examined whether pre-operative ctDNA levels in 119 patients with stage III (B-D) melanoma was associated with outcome following surgery. CtDNA was detected in pre-operative samples of $34 \%$ and $33 \%$ of patients in their discovery and validation cohorts, respectively. The time to distant metastatic relapse
(DMFS) was shorter in patients with detectable ctDNA with a median of 6.2 months vs. 13.9 months (HR 1.59; 95\% CI $1.0-2.52 ; P=0.027)$ and 8.7 months vs. 14.5 months (HR 2.15; 95\% CI 1.04-4.47, $P=0.014)$, in the discovery and validation cohorts, respectively [13•]. In addition, patients with detectable ctDNA had a median melanoma-specific survival (MSS) of 17.6 months compared with 49.4 months in those with undetectable levels (HR 2.11; 95\% CI 1.20-3.71, $P<0.01$ ) [13•]. This remained significant in multivariable analysis of characteristics including stage, number of lymph nodes, size of lymph nodes, and extranodal extension [13•]. All these studies used droplet digital PCR (ddPCR) to identify ctDNA in volumes of up to $5 \mathrm{ml}$ of plasma. Based on these data, there is a potential role for ctDNA in augmenting current staging methods in order to predict survival of patients with stage III melanoma. Furthermore, results from ctDNA analyses expected to be reported from these pivotal trials may inform as to its role in predicting risk of recurrence, survival and response to adjuvant treatment.

Through serial assessments of ctDNA, it is possible to identify MRD and early disease relapse (molecular progression) following curative intent surgery for early stage melanoma. In a retrospective study of 161 patients with stage III melanoma and no radiological evidence of disease, detection of BRAF/NRAS mutant ctDNA (by ddPCR) at a single timepoint within 12 weeks of surgery was associated with poor outcome (disease-free Interval (DFI); HR 3.12; 95\% CI 1.79-5.47; $P<0.0001$, distant metastasis-free interval (DMFI); HR 3.22; 95\% CI 1.80-5.79; $P<0.0001$, OS HR $2.63 ; 95 \%$ CI $1.40-4.96 ; P=0.003$ ) [15••]. The presence of ctDNA remained a significant predictor of relapse and OS in multivariate analyses $[15 \bullet \bullet$. These results were later vali-

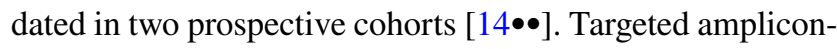
based next-generation sequencing (NGS) or pyrosequencing were used to identify mutations in formalin-fixed-paraffinembedded (FFPE) tissue in order to develop bespoke ddPCR assays for specific point mutations. Detection of ctDNA within 12 weeks of surgery was a strong predictor of relapse (RFS HR 10; 95\% CI 4.3-24; $P<0.001$ ), with all but one patient across the two cohorts experiencing disease progression within the 20-month follow-up period [14••]. Critically, longitudinal monitoring improved the sensitivity of detecting relapse over time, with a number of patients who were negative at the first post-operative timepoint subsequently developing detectable ctDNA prior to relapse [14••]. There remains a challenge in the use of ctDNA to inform a decision to give adjuvant therapy immediately post-operatively as there may be false negative results with patients subsequently relapsing. Technical improvements in sensitivity of the assays may aid future decision-making at this timepoint, or longitudinal monitoring may offer an alternative strategy.

Evaluation of ctDNA in patients may also inform monitoring of response to adjuvant ICI following curative surgery. In a small study of 18 patients who received adjuvant ICI 
(anti-PD-1), 4/14 patients had detectable ctDNA post-operatively, and in 2 of these patients, ctDNA subsequently become undetectable during treatment with ICI [14••]. Critically, after 7 months of follow-up, there was no evidence of relapse, suggesting that ctDNA could be used to define early response to adjuvant treatment. Larger studies and longer-term follow-up are required to validate these results.

Based on retrospective and prospective data demonstrating that ctDNA could be used to longitudinally monitor for molecular recurrence in the post-operative setting, we developed the DETECTION phase II/III study (Circulating tumour DNA guidEd Therapy for stage IIB/C mElanoma after surgiCal resection; NCT04901988). The study aims to address a challenge in managing patients with stage II melanoma where the individual risk of death for patients with stage IIB/C melanoma is low, but because they are very common, they contribute to $30-50 \%$ of all melanoma deaths $[16,17]$. CheckMate 76K (NCT04099251) and Keynote 716 (NCT03553836) are examining the role of adjuvant anti-PD-1 in preventing relapse of stage IIB/C melanoma. However, treating unselected patients upfront exposes a large number to toxicity and potential impact on quality of life, when their disease is already cured by surgery alone. In addition, there is a financial and resource cost to the health system. DETECTION uses ctDNA to monitor for MRD and molecular relapse following surgery for stage IIB/C melanoma. If ctDNA is detected, patients are randomised to standard of care, which is treatment upon clinical/radiological relapse or early treatment with nivolumab. It will prospectively assess whether ctDNA can identify disease recurrence earlier than standard of care and critically whether early treatment of micro-metastatic disease with ICI improves outcomes.

\section{Advanced Melanoma}

CtDNA can also be used to guide treatment choice and monitor response in the setting of stage IV disease. A number of studies in advanced disease have shown that a decline in plasma ctDNA is associated with response and may often precede radiological evidence of progression [18, 19, 20•, $21,22]$. Levels of cfDNA have been shown to correlate with tumour burden on CT scan and levels of ctDNA have been shown to correspond with metabolic burden of disease as assessed by FDG-PET [20•, 23, 24]. However, there may be variation in ctDNA release according to disease site with patients with visceral, bone, or lymph node involvement exhibiting higher levels of ctDNA out of keeping with the metabolic disease burden as assessed by FDG-PET, whilst those with extensive subcutaneous disease or with brain metastases showed consistently low levels of ctDNA despite measurable disease [20•].
However, there is huge potential for ctDNA to be used as a tool to guide treatment decisions for patients receiving both targeted agents and ICI.

\section{Targeted Therapy}

Targeted therapy with combination BRAF and MEK inhibitors is a key line of treatment in patients with advanced $B R A F$ V600 mutant melanoma. Based on data from clinical trials showing improvement in both PFS and OS, these inhibitors are now approved as standard of care and are widely used in management of patients with stage IV melanoma [25-27]. A number of studies have shown that ctDNA can be used at baseline as a predictive biomarker of response to targeted therapy, an on-treatment biomarker of response/disease progression and a tool to identify mechanisms of resistance.

The baseline level of mutant $B R A F$ in cfDNA has been shown to be a predictive biomarker of duration of therapy in patients treated with the BRAF/MEK inhibitors. In a pooled analysis from four clinical trials, enrolling patients with tissue confirmed $B R A F$ V600E/K mutant melanoma; copies of mutant $B R A F$ were identified in $76 \%$ (V600E) and $81 \%$ (V600K) of 732 baseline plasma samples using BEAMing (beads, emulsion, amplification). Its presence was associated with lower response rates compared to those patients where mutant $B R A F$ was undetectable [19]. Furthermore, patients who had detectable mutant $B R A F$ had a significantly shorter PFS and OS compared to those with undetectable levels [28]. This remained significant in multivariable analysis comparing baseline factors including lactase dehydrogenase (LDH) and performance status in 3 out of 4 of the studies [28]. More recently, a further validation study of patients treated in the COMBI$\mathrm{d}$ and COMBI-MB trials of dabrafenib plus trametinib (COMBI-MB in patients with brain metastases) supported baseline detectable ctDNA as a predictor of PFS and OS on targeted therapy [29•]. Using a cut-off of 64 copies of ctDNA per millilitre (determined using ddPCR), they stratified patients as high and low risk. Patients with lowvs. high-risk disease had a significantly longer PFS 12.7 vs. 6.5 months (HR 1.74; 95\% CI 1.37-2.21, $p<0.0001$ ) and 35.1 vs. 13.4 months (HR 2.23; 95\% CI 1.73-2.87, $p<0.0001$ ) [29•].

There is also increasing evidence that ctDNA could be used to monitor patients on therapy, and that ctDNA changes may be detectable ahead of radiological or biochemical lactate dehydrogenase (LDH) change, giving an early indicator as to treatment efficacy or an early herald of disease progression. A study compared ctDNA vs. LDH in identifying disease progression within 15 days of confirmed radiological progression in 26 patients. In $82 \%$ of 
patients, ctDNA progression was detected vs. only $40 \%$ having an LDH rise, with a median difference in sensitivity of $42 \%$ (95\% confidence interval, $27-58 \% ; p<0.001$ ) [30]. A further study took longitudinal samples from 36 patients on targeted therapy, with 12 of these having detectable ctDNA levels prior to commencement of treatment [31]. There was a significant decrease in ctDNA in all of the patients $(p<0.01)$, with median time to becoming undetectable $(n=7)$ or $<1 \%(n=5)$ of 13 days (range 6-40 days) [31]. An increase in the BRAF V600 mutant ctDNA fraction was detected prior to the clinical diagnosis of progressive disease in 12 out of 27 (44\%) patients and simultaneously with PD in 7 out of 27 (26\%) patients [31]. These data were subsequently confirmed in another study, which showed that ctDNA levels increased before radiological progression by a mean of 110 days [32].

Although one study did not support the use of ctDNA as an on-treatment biomarker of response to targeted therapy [31], larger studies have shown that it could be a useful tool [29•, 33]. One study investigated whether ctDNA levels of 10 copies per $\mathrm{mL}$ or higher in the first plasma sample since treatment initiation were predictive of PFS. They found that patients with undetectable ctDNA had a median PFS of 9 vs. 4 months in patients with detectable ctDNA (HR, 4.05; 95\% CI, 1.56 to 10.53). A large analysis from the COMBI-d trial showed that 201/224 patients had detectable ctDNA at baseline, and 121/201 still had detectable ctDNA following 4 weeks of treatment. Disease control (complete response, partial response or stable disease) as best overall response was associated with $65 / 80$ patients with conversion to undetectable levels vs. $63 / 118$ patients with detectable ctDNA at week 4 (proportional-odds-likelihood-ratio test for association $P=0.0002$ ) [29•]. In patients with high LDH only (above upper limit of normal), undetectable ctDNA at 4 weeks was significantly associated with PFS (HR 1.99; 95\% CI 1.08-3.64, $p=0.027$ ) and OS (HR 2.38; 95\% CI 1.24-4.54, $p=0.0089$ ) [29•]. Conversion to undetectable ctDNA at week 4 was independently associated with both PFS and OS in a Cox regression model that included clinical prognostics factors such as performance status and 3 or more organ sites with metastases [29•]. Taken together, these studies suggest that decreasing ctDNA levels on treatment are predictive of response to targeted therapy.

Based on the understanding that ctDNA could be used as a sensitive biomarker of response and progression on therapy, the DyNAMIc trial (circulating tumour DNA guided adaptive BRAF and MEK inhibitor therapy) has been developed. This is a pilot study testing feasibility of and examining whether adaptive therapy could improve PFS in patients on targeted therapy $(\mathrm{E}+\mathrm{B})$. Adaptive therapy relies on the competition between drug-sensitive and drug-resistant subclones to control overall tumour growth $[34,35]$. It aims to stabilise tumour burden by allowing a significant population of treatment-sensitive cells to survive, which suppress proliferation of the less fit, resistant populations [34]. In vivo, this was shown to be more effective than intermittent scheduling [36], which was not shown to be beneficial in SWOG S1320 (median 9.0 months continuous vs. 5.5 months intermittent, $P=0.064$, pre-specified two-sided $\alpha=0.2)$ [37], although results from INTERIM (NCT03352947), which uses different timing of scheduling to SWOG S1320, are awaited. DyNAMIc will use the number of mutant $B R A F$ copies $/ \mathrm{ml}$ in cfDNA to determine when to stop and start drugs based on pre-specified thresholds which will be optimised in the trial.

Finally, a number of studies have shown that ctDNA can identify mechanisms of resistance to therapy. Studies have shown the emergence of NRAS, MAP2K1, AKTl, and $P I K 3 C A$ mutations in patients treated with targeted therapy, which are known to be mechanisms of resistance to MAPK targeting therapy in melanoma and were associated with subsequent disease progression identified on CT scan [20•, 21, 38]. More recently, ARAF mutations, shown to be a novel mechanism of resistance to the selective RAF dimer (type II) inhibitor belvarafenib, have been described [39]. Therefore, ctDNA can be used to identify mechanisms of resistance, which could potentially aid patient selection or next-line therapy options.

\section{Immunotherapy}

ICI have played a vital role in transforming clinical management and outlook of patients with melanoma. On the basis of data from randomised studies showing OS benefit, they are used as standard care treatment of metastatic melanoma and have more recently been shown to improve PFS in the adjuvant setting with OS data awaited $[8,10]$. Both anti-PD- 1 as single agents and combination anti-PD-1 plus anti-CTLA-4 inhibitors are currently used in the clinic. The combination of nivolumab plus ipilimumab $(\mathrm{N}+\mathrm{I})$ results in increased depth of response compared to single agents, however, is associated with increased toxicity. More recently, the RELATIVITY-047 Phase III trial showed improved PFS when relatlimab a LAG-3 antibody was combined with nivolumab vs. nivolumab alone [40]. Further ICI combinations are currently in early phase trials, and therefore in future, a key focus will be how to optimise sequencing of these therapies to extend PFS and OS. CtDNA may be a tool that could facilitate better decisions as to when to switch therapy.

Due to the heterogeneity of response to ICI, one of the main challenges is to identify predictive biomarkers, which could enable tailoring of treatment for the individual. A number of studies have shown that ctDNA can be used as a baseline biomarker of response to ICI [41, 42, 43••, 44, 45]. One study in 19 patients with $B R A F$ and NRAS mutations showed that a baseline ctDNA level of $<10$ copies $/ \mathrm{ml}$ 
plasma prior to commencing ICI (anti-CTLA-4/anti-PD-1/ combination) was associated with a significantly longer PFS $(P=0.009$, relative risk $=5 ; 95 \%$ CI 1.8-13.8) [38]. In support of these findings, a further study in 141 patients treated with pembrolizumab found that patients with undetectable ctDNA at baseline vs. those with detectable levels of ctDNA using real-time PCR (BRAF) or ddPCR (NRAS) in $1 \mathrm{ml}$ of plasma had a median PFS of 26 vs. 9 weeks (HR 0.47; $P=0.01)$ and $\mathrm{OS}$ not reached vs. 21.3 weeks $(\mathrm{HR}=0.37$; $P=0.005$ ) [41]. This remained significant in multivariable analysis including LDH, CRP, number of metastatic sites $(>3)$, and performance status. Intriguingly, a recent study showed that baseline ctDNA appears to only be a biomarker of response in the first-line setting [44]. In two (albeit small) independent cohorts of patients with the majority receiving targeted therapy first line, baseline ctDNA of 20 copies $/ \mathrm{ml}$ identified using ddPCR was predictive of longer PFS in first line but not second line in patients treated with ICI [44]. Whether this is the same if treated with ICI first line with new combinations such as anti-LAG3 plus anti-PD-1 will require further studies.

CtDNA has also been shown to be an on-treatment biomarker of response to ICI. Initially, rising ctDNA levels during longitudinal sampling of patients receiving ICI were shown to correlate with disease progression [42]. Subsequent studies have shown that clearance of ctDNA on treatment is a biomarker of PFS and OS [41, 43••, 45]. One study examined whether undetectable ctDNA levels (in $\geq 1 \mathrm{ml}$ plasma using ddPCR) at baseline (group A, $n=36$ ) and clearance of ctDNA from initially detectable baseline levels within 12 weeks of treatment with PD-1 antibodies (group B, $n=22$ ) compared to those patients with persistently elevated ctDNA (group C, $n=18$ ) were associated with response [45]. The median PFS was not reached in groups A and B and was 2.7 months for group $\mathrm{C}(P<0.001$, HR 0.09 for group A vs. C, and $P<0.001$ HR 0.16 for group $\mathrm{B}$ vs. C). The median OS was not reached for groups $\mathrm{A}$ and $\mathrm{B}$ and was 9.2 months for group $\mathrm{C}(P<0.001, \mathrm{HR} 0.02$ for group A vs. $\mathrm{C}$ and $P<0.0010 .14$; for group $\mathrm{B}$ vs. $\mathrm{C})$. This remained significant in multi-variable analysis of clinical features including LDH, performance status, tumour stage and disease volume. This is supported by another study in 85 patients (in $1 \mathrm{ml}$ plasma using rtPCR and ddPCR), which showed that patients with undetectable ctDNA levels during follow-up (median 84 weeks) had improved survival compared to those with ctDNA present (adjusted HR for death 0.16 ; 95\% CI $0.07-0.36, P<0.001$ ) [41]. A further study demonstrated that ctDNA dynamics as early as 2 weeks may be predictive of clinical response [43••]. No biological response defined as no reduction of ctDNA from baseline (alpha 2.5\%) at 2 weeks was associated with a $0 \%$ response rate (0/10 patients) and a 0\% PFS rate at 120 days (median $\mathrm{PFS}=112$ days) and a median OS of 130 days. In addition, all those who had a significant increase of ctDNA following an initial decline were found to have radiological progression at an average of 75 days in advance of radiologic detec-

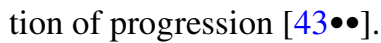

CtDNA could also be a useful tool to clarify response in pseudo-progression, which is defined as an increase in the size of the primary tumour or the appearance of a new lesion followed by tumour regression, a phenomenon seen in approximately $10-30 \%$ of patients treated with ICI in melanoma [46, 47]. A favourable ctDNA profile (undetectable at baseline or conversion to undetectable by week 12) was able to differentiate true progression from pseudo-progression with a sensitivity of $90 \%$ (95\% CI 68-99\%) and specificity of $100 \%$ (95\% CI 66-100\%), which had better discrimination compared to LDH [48•]. This suggests that ctDNA is not simply a measure of tumour burden, but also reflects underlying tumour-immune response. The ability to distinguish pseudo-progression from true progression could reduce the need for repeat imaging and may prevent treatment being discontinued unnecessarily.

Moving towards using ctDNA as a potential tool to guide clinical decisions, the Circulating Tumour DNA Guided Switch (CAcTUS; NCT03808441) phase II feasibility trial in patients with $B R A F$ mutant melanoma is examining whether response to ICI can be improved through pre-treatment with targeted therapy and switching treatment in response rather than resistance [49••]. Tumours responding to BRAF/MEK inhibitors have been shown to have increased $\mathrm{T}$ cell infiltration, improved $\mathrm{T}$ cell recognition of melanoma associated antigens and reduced production of immunosuppressive cytokines [50-53]. The SECOMBIT (NCT02631447) and EBIN (NCT03235245) studies are both assessing whether a pre-specified period of induction targeted therapy (12 weeks for EBIN and 8 weeks for SECOMBIT) results in improved survival $[54,55]$. However, these strategies do not personalise treatment to the individual response of a patient and are at risk (particularly with 12 weeks of therapy) of patients already developing resistant clones which are more immune suppressive. CAcTUS personalises the decision to switch based on response seen in ctDNA. Patients on the intervention arm receive targeted therapy until there is evidence of response as defined by a decrease in mutant BRAF variant allele frequency (VAF) of $\geq 80 \%$ measured by ddPCR. The primary endpoint is logistical and assesses whether ctDNA results can be provided within 7 days with secondary endpoints providing a signal search as to efficacy of the strategy and insight into ctDNA monitoring.

\section{Central Nervous System Disease}

Although ctDNA has been shown to be effective in identifying early disease progression, caution must be taken when assessing central nervous system (CNS) disease. A number 
of studies have reported undetectable ctDNA in patients with CNS-only metastases [23, 29•, 32, 56, 57, 58••]. In addition, the ability of plasma ctDNA to detect CNS progression (especially if extra-cranial disease is stable) has been shown to be variable, with one group reporting ctDNA progression associated with incidence of new brain metastases in 10/12 (83\%) patients [18], whilst others have shown discordance in radiological and ctDNA progression [32]. Undetectable ctDNA on-therapy was associated with extracranial response $(P<0.01)$ but not intracranial response [58••]. This is an important consideration for on-treatment assessments where CNS-only progression may be missed, and also in the monitoring for early disease relapse.

CtDNA in CSF may provide an alternative tool for detection of CNS-only disease and has been shown to be more sensitive than plasma ctDNA in this context [59-61]. A number of case reports have suggested that CSF ctDNA may be useful for monitoring intracranial disease response or identifying targetable mutations, but there have not been any large studies performed in melanoma to validate these findings [62, 63]. In addition, CSF ctDNA may be useful in the diagnosis of leptomeningeal disease where radiological diagnosis can be challenging and cytological material in the CSF may be limited or even where present may be insufficient to permit analysis for $B R A F$ mutations [64]. Due to the invasive nature of lumbar puncture, it is unlikely that ctDNA in CSF will be used for treatment monitoring; however, it may be useful to identify targetable mutations both at baseline and on progression and could be used as a predictor of future CNS disease.

\section{Conclusions}

CtDNA is a powerful tool which has the potential to support tailored management of patients with melanoma throughout their treatment journey. By offering an accurate, easily obtainable method of assessing a patient's disease status, ctDNA could enable precise and timely treatment decisions to be made which optimise efficacy and minimise unnecessary treatment burden. The potential for longitudinal sampling means that ctDNA can offer an early and dynamic assessment of treatment response. CtDNA can also characterise intra-tumour heterogeneity and differences in clonal response to therapy. Liquid biopsies are less invasive than tissue biopsies and therefore are useful if patients have disease in locations inaccessible/at high risk of complications. However, in general, ctDNA can only provide information on mutational or copy number changes, although new methods to infer gene expression based on nucleosome occupancy are being developed [65]. Other types of liquid biopsies such as CTCs used in parallel could give additional information regarding gene and protein expression.
In this review, we have highlighted a number of different potential applications for ctDNA. However, there are a number of challenges to overcome if ctDNA is to move into the clinic and be used to guide treatment decisions. There is anatomical variability in the extent of ctDNA release from particular sites including subcutaneous and intracranial disease, which may limit its utility in for certain patients [20•]. In addition, identifying MRD in particular can be technically challenging, although newer approaches using size selection to reduce background noise [4], improving bioinformatics pipelines [66], multiplexing [67] or analysing for methylation changes to increase the chances of identifying aberrations compared to normal cfDNA [68] could all improve detection. In addition, clonal haematopoiesis can also result in false positive results especially in genes associated with epigenetic modulators such as DNMT3A as well as TP53; therefore, some results may need to be interpreted with caution [69-71].

Over the past 10 years, a huge number of techniques have been developed to analyse ctDNA. However, there is currently no standardised approach to compare them, which makes future-proofing clinical trials a challenge. Furthermore, though assays may be technically better at identifying ctDNA, their clinical benefit may be minimal. For example, assay A may improve detection of MRD in the laboratory, however may only detect disease recurrence 2 weeks before assay B in a patient, which is unlikely to result in clinical benefit. As a field, standards need to be developed and agreed upon to use for assay comparison. In addition, particularly in the context of MRD detection, there needs to be discussion and a framework developed as to how newer (e.g. more sensitive) assays could be substituted if proof-of principal for improved patient outcome was established with older assays.

Finally, although there have been many potential uses for ctDNA identified pre-clinically, it is critical that its clinical benefit is established using randomised-controlled trials. Being able to identify early progression or treatment resistance does not necessarily mean that treating early or switching early to a new treatment line will improve outcomes for the patient. Lessons can be learnt from the SWOG S0500 trial, which did not show OS improvement for patients with breast cancer with persistently increased CTCs after 21 days of first-line chemotherapy who were switched early to an alternative treatment and OV05/EORTC 5595, which did not show a survival benefit for early treatment of relapse on the basis of a raised CA125 [72,73]. Thus the next stage of testing of ctDNA within clinical trials is crucial to ensure that ctDNA is optimally used to improve outcomes for patients with cancer.

CtDNA has huge potential to provide real-time information about tumour activity, disease status, treatment response/progression and mutational profile at any given 
time following melanoma diagnosis. It is now time to extensively test its value within the clinic in order to improve patient outcomes.

\section{Declarations}

Conflict of Interest Ann Tivey is supported by the National Institute for Health Research (NIHR) as an NIHR Academic Clinical Fellow. Fiona Britton declares that she has no conflict of interest.

Julie-Ann Scott declares that she has no conflict of interest.

Dominic Rothwell declares that she has no conflict of interest.

Paul Lorigan has received clinical trial support from Bristol-Myers Squibb, Novartis, GlaxoSmithKline and Amgen; has received speaker's honoraria from Bristol-Myers Squibb, Merck, GlaxoSmithKline, Chugai Pharmaceutical Co., Ltd, Laboratoires Pierre Fabre, NeraCare, Roche and Oncology Education Canada; and has received compensation for participation on advisory boards from Bristol-Myers Squibb, Merck, Novartis, GlaxoSmithKline, Amgen, Chugai Pharmaceutical Co., Ltd, Laboratoires Pierre Fabre, NeraCare, Roche and Oncology Education Canada.

Rebecca Lee has received research funding from Bristol-Myers Squibb for the CAcTUS Trial, and has received speaker's honoraria from AstraZeneca.

Open Access This article is licensed under a Creative Commons Attribution 4.0 International License, which permits use, sharing, adaptation, distribution and reproduction in any medium or format, as long as you give appropriate credit to the original author(s) and the source, provide a link to the Creative Commons licence, and indicate if changes were made. The images or other third party material in this article are included in the article's Creative Commons licence, unless indicated otherwise in a credit line to the material. If material is not included in the article's Creative Commons licence and your intended use is not permitted by statutory regulation or exceeds the permitted use, you will need to obtain permission directly from the copyright holder. To view a copy of this licence, visit http://creativecommons.org/licenses/by/4.0/.

\section{References}

Papers of particular interest, published recently, have been highlighted as:

- Of importance

$\bullet$ Of major importance

1. Schwarzenbach H, Hoon DSB, Pantel K. Cell-free nucleic acids as biomarkers in cancer patients. Nat Rev Cancer. 2011;11(6):426-37.

2. Szpechcinski A, Chorostowska-Wynimko J, Struniawski R, Kupis W, Rudzinski P, Langfort R, et al. Cell-free DNA levels in plasma of patients with non-small-cell lung cancer and inflammatory lung disease. Br J Cancer. 2015;113(3):476-83.

3. Choi J, Reich CF, Pisetsky DS. The role of macrophages in the in vitro generation of extracellular DNA from apoptotic and necrotic cells. Immunology. 2005;115(1):55-62.

4. Mouliere F, Chandrananda D, Piskorz AM, Moore EK, Morris J, Ahlborn LB, et al. Enhanced detection of circulating tumor DNA by fragment size analysis. Sci Transl Med. 2018;10(466):eaat4921.
5.• Sorenson G, Pribish D, Valone F, Memoli V, Bzik D, Yao S. Soluble normal and mutated DNA sequences from single-copy genes in human blood. Cancer Epidemiol Biomarkers Prev. 1994;3(1):67-71. First study to report the utility of ctDNA for cancer detection.

6. Keller L, Belloum Y, Wikman H, Pantel K. Clinical relevance of blood-based ctDNA analysis: mutation detection and beyond. Br J Cancer. Springer Nature. 2021;124:345-58.

7. Pantel K, Alix-Panabières C. Liquid biopsy and minimal residual disease - latest advances and implications for cure. Nat Rev Clin Oncol. Nature Publishing Group. 2019;16:409-24.

8. Hauschild A, Dummer R, Schadendorf D, Santinami M, Atkinson $\mathrm{V}$, Mandalà $\mathrm{M}$, et al. Longer follow-up confirms relapsefree survival benefit with adjuvant dabrafenib plus trametinib in patients with resected BRAF V600-mutant stage III melanoma. J Clin Oncol. 2018;36(35):3441-9.

9. Eggermont AMM, Chiarion-Sileni V, Grob J-J, Dummer R, Wolchok JD, Schmidt H, et al. Prolonged survival in stage III melanoma with ipilimumab adjuvant therapy. N Engl J Med. 2016;375(19):1845-55.

10. Ascierto PA, Minor D, Ribas A, Lebbe C, O'Hagan A, Arya N, et al. Phase II trial (BREAK-2) of the BRAF inhibitor dabrafenib (GSK2118436) in patients with metastatic melanoma. J Clin Oncol [Internet]. 2013 Sep 10 [cited 2021 Apr 9];31(26):320511. Available from: http://ascopubs.org/doi/10.1200/JCO.2013. 49.8691.

11. Dummer R, Hauschild A, Santinami M, Atkinson V, Mandalà M, Kirkwood JM, et al. Five-year analysis of adjuvant dabrafenib plus trametinib in stage III melanoma. N Engl J Med. 2020;383(12):1139-48.

12. Higham CE, Chatzimavridou-Grigoriadou V, Fitzgerald CT, Trainer PJ, Eggermont AMM, Lorigan P. Adjuvant immunotherapy: the sting in the tail. Eur J Cancer. 2020;132:207-10.

13. Lee JH, Saw RP, Thompson JF, Lo S, Spillane AJ, Shannon KF, et al. Pre-operative ctDNA predicts survival in high-risk stage III cutaneous melanoma patients. Ann Oncol. 2019;30(5):815-22. This study demonstrated that pre-operative ctDNA levels can predict melanoma-specific survival in patients with highrisk stage III melanoma undergoing lymph node dissection, potentially guiding use of (neo)adjuvant therapy.

14.• Tan L, Sandhu S, Lee RJ, Li J, Callahan J, Ftouni S, et al. Prediction and monitoring of relapse in stage III melanoma using circulating tumor DNA. Ann Oncol [Internet]. 2019 May 1 [cited 2021 Apr 9];30(5):804-14. Available from: https://pubmed.ncbi. nlm.nih.gov/30838379/. This study showed that baseline and postoperative ctDNA detection in two independent prospective cohorts could identify patients with stage III melanoma at highest risk of relapse. In addition, it showed that longitudinal monitoring following surgical resection increased sensitivity. This could potentially inform monitoring of patients following surgery.

15.• Lee RJ, Gremel G, Marshall A, Myers KA, Fisher N, Dunn JA, et al. Circulating tumor DNA predicts survival in patients with resected high-risk stage II/III melanoma. Ann Oncol. 2018;29(2):490-6. First study to show that detection of BRAF/NRAS mutations in ctDNA following surgical resection predicts for relapse and survival in stage II/III melanoma; this indicates its potential value in informing adjuvant therapy decisions.

16. Whiteman DC, Baade PD, Olsen CM. More people die from thin melanomas $(<1 \mathrm{~mm})$ than from thick melanomas $(>4 \mathrm{~mm})$ in Queensland, Australia. J Invest Dermatol. 2015;135(4):1190-3.

17. Gershenwald JE, Scolyer RA, Hess KR, Sondak VK, Long GV, Ross MI, et al. Melanoma staging: evidence-based changes in the American Joint Committee on Cancer eighth edition cancer staging manual. CA Cancer J Clin. 2017;67(6):472-92. 
18. Chang-Hao Tsao S, Weiss J, Hudson C, Christophi C, Cebon J, Behren A, et al. Monitoring response to therapy in melanoma by quantifying circulating tumour DNA with droplet digital PCR for BRAF and NRAS mutations. Sci Rep. 2015;5(1):11198.

19. Santiago-Walker A, Gagnon R, Mazumdar J, Casey M, Long $\mathrm{GV}$, Schadendorf D, et al. Correlation of BRAF mutation status in circulating-free DNA and tumor and association with clinical outcome across four BRAFi and MEKi clinical trials. Clin Cancer Res. 2015;1-9.

20. Wong SQ, Raleigh JM, Callahan J, Vergara IA, Ftouni S, Hatzimihalis A, et al. Circulating tumor DNA analysis and functional imaging provide complementary approaches for comprehensive disease monitoring in metastatic melanoma. JCO Precis Oncol. 2017;(1):1-14. Study demonstrating concordance of ctDNA levels and metabolic tumour burden, but also highlighting the differences in ctDNA levels between anatomical sites.

21. Girotti MR, Gremel G, Lee R, Galvani E, Rothwell D, Viros A, et al. Application of sequencing, liquid biopsies, and patientderived xenografts for personalized medicine in melanoma. Cancer Discov. 2016;6(3):286-99.

22. Gremel G, Lee RJ, Girotti MR, Mandal AK, Valpione S, Garner $\mathrm{G}$, et al. Distinct subclonal tumour responses to therapy revealed by circulating cell-free DNA. Ann Oncol [Internet]. 2016 Oct 1 [cited 2021 May 7];27(10):1959-65. Available from: http://www. annalsofoncology.org/article/S0923753419359071/fulltext.

23. McEvoy AC, Warburton L, Al-Ogaili Z, Celliers L, Calapre L, Pereira MR, et al. Correlation between circulating tumour DNA and metabolic tumour burden in metastatic melanoma patients. BMC Cancer. 2018;18(1):1-8.

24. Valpione S, Gremel G, Mundra P, Middlehurst P, Galvani E, Girotti MR, et al. Plasma total cell-free DNA (cfDNA) is a surrogate biomarker for tumour burden and a prognostic biomarker for survival in metastatic melanoma patients. Eur J Cancer. 2018;88:1-9.

25. Long GV, Stroyakovskiy D, Gogas H, Levchenko E, de Braud F, Larkin J, et al. Combined BRAF and MEK inhibition versus BRAF inhibition alone in melanoma. N Engl J Med. 2014;371(20):1877-88.

26. Dummer R, Ascierto PA, Gogas HJ, Arance A, Mandala M, Liszkay G, et al. Encorafenib plus binimetinib versus vemurafenib or encorafenib in patients with BRAF -mutant melanoma (COLUMBUS): a multicentre, open-label, randomised phase 3 trial. Lancet Oncol. 2018;19(5):603-15.

27. Larkin J, Ascierto PA, Dréno B, Atkinson V, Liszkay G, Maio M, et al. Combined vemurafenib and cobimetinib in $B R A F$-mutated melanoma. N Engl J Med. 2014;371(20):1867-76.

28. Santiago-Walker A, Gagnon R, Mazumdar J, Casey M, Long $\mathrm{GV}$, Schadendorf D, et al. Correlation of BRAF mutation status in circulating-free DNA and tumor and association with clinical outcome across four BRAFi and MEKi clinical trials. Clin Cancer Res. 2015;1-9.

29.• Syeda MM, Wiggins JM, Corless BC, Long GV, Flaherty KT, Schadendorf D, et al. Circulating tumour DNA in patients with advanced melanoma treated with dabrafenib or dabrafenib plus trametinib: a clinical validation study. Lancet Oncol. 2021;22(3):370-80. The largest validation study to date demonstrating pre-treatment and on-treatment BRAFV600mutant ctDNA measurements in patients with late stage melanoma receiving targeted therapy were predictive for progression-free and overall survival.

30. Chang GA, Tadepalli JS, Shao Y, Zhang Y, Weiss S, Robinson E, et al. Sensitivity of plasma BRAFmutant and NRASmutant cellfree DNA assays to detect metastatic melanoma in patients with low RECIST scores and non-RECIST disease progression. Mol Oncol [Internet]. 2016 Jan 1 [cited 2021 May 10];10(1):157-65. Available from: https://doi.org/10.1016/j.molonc.2015.09.005.
31. Schreuer M, Meersseman G, Van Den Herrewegen S, Jansen Y, Chevolet I, Bott A, et al. Quantitative assessment of BRAF V600 mutant circulating cell-free tumor DNA as a tool for therapeutic monitoring in metastatic melanoma patients treated with BRAF/ MEK inhibitors. J Transl Med. 2016;14(1):95.

32. Haselmann V, Gebhardt C, Brechtel I, Duda A, Czerwinski C, Sucker A, et al. Liquid profiling of circulating tumor DNA in plasma of melanoma patients for companion diagnostics and monitoring of BRAF inhibitor therapy. Clin Chem. 2018;64(5):830-42.

33. Braune J, Keller L, Schiller F, Graf E, Rafei-Shamsabadi D, Wehrle J, et al. Circulating tumor DNA allows early treatment monitoring in BRAF- and NRAS-mutant malignant melanoma. JCO Precis Oncol. 2020;4:20-31.

34. Zhang J, Cunningham JJ, Brown JS, Gatenby RA. Integrating evolutionary dynamics into treatment of metastatic castrateresistant prostate cancer. Nat Commun. 2017;8(1):1816.

35. West JB, Dinh MN, Brown JS, Zhang J, Anderson AR, Gatenby RA. Multidrug cancer therapy in metastatic castrate-resistant prostate cancer: an evolution-based strategy. Clin Cancer Res. 2019;25(14):4413-21.

36. Smalley I, Kim E, Li J, Spence P, Wyatt CJ, Eroglu Z, et al. Leveraging transcriptional dynamics to improve BRAF inhibitor responses in melanoma. EBioMedicine. 2019.

37. Algazi AP, Othus M, Daud AI, Lo RS, Mehnert JM, Truong TG, et al. Continuous versus intermittent BRAF and MEK inhibition in patients with BRAF-mutated melanoma: a randomized phase 2 trial. Nat Med. 2020;26(10):1564-8.

38. Gray ES, Rizos H, Reid AL, Boyd SC, Pereira MR, Lo J, et al. Circulating tumor DNA to monitor treatment response and detect acquired resistance in patients with metastatic melanoma. Oncotarget. 2015;6(39):42008-18.

39. Yen I, Shanahan F, Lee J, Hong YS, Shin SJ, Moore AR, et al. ARAF mutations confer resistance to the RAF inhibitor belvarafenib in melanoma. Nature. 2021;1-6.

40. Lipson EJ, Tawbi HA-H, Schadendorf D, Ascierto PA, Matamala L, Gutiérrez EC, et al. Relatlimab (RELA) plus nivolumab (NIVO) versus NIVO in first-line advanced melanoma: primary phase III results from RELATIVITY-047 (CA224-047). J Clin Oncol [Internet]. 2021 May 20 [cited 2021 Jun 7];39(15_ suppl):9503-9503. Available from: https://ascopubs.org/doi/10. 1200/JCO.2021.39.15_suppl.9503

41. Seremet T, Jansen Y, Planken S, Njimi H, Delaunoy M, El HH, et al. Undetectable circulating tumor DNA (ctDNA) levels correlate with favorable outcome in metastatic melanoma patients treated with anti-PD1 therapy. J Transl Med. 2019;17:303.

42. Lipson EJ, Velculescu VE, Pritchard TS, Sausen M, Pardoll DM, Topalian SL, et al. Circulating tumor DNA analysis as a real-time method for monitoring tumor burden in melanoma patients undergoing treatment with immune checkpoint blockade. J Immunother cancer. 2014;2(1):42.

43.• Herbreteau G, Vallée A, Knol AC, Théoleyre S, Quéreux G, Varey E, et al. Quantitative monitoring of circulating tumor DNA predicts response of cutaneous metastatic melanoma to anti-PD1 immunotherapy. Oncotarget [Internet]. 2018 May 18 [cited 2021 May 10];9(38):25265-76. Available from: /pmc/ articles/PMC5982743/. Study demonstrating that ctDNA can detect progression earlier than imaging when assessing metastatic melanoma treated with anti-PD-1 therapy.

44. Marsavela G, Lee J, Calapre L, Wong SQ, Pereira MR, McEvoy AC, et al. Circulating tumor DNA predicts outcome from first-, but not second-line treatment and identifies melanoma patients who may benefit from combination immunotherapy. Clin Cancer Res. 2020;26(22):5926-33.

45. Lee JH, Long GV, Boyd S, Lo S, Menzies AM, Tembe $\mathrm{V}$, et al. Circulating tumour DNA predicts response to 
anti-PD1 antibodies in metastatic melanoma. Ann Oncol. 2017;28(5):1130-6.

46. Pires da Silva I, Lo S, Quek C, Gonzalez M, Carlino MS, Long $\mathrm{GV}$, et al. Site-specific response patterns, pseudoprogression, and acquired resistance in patients with melanoma treated with ipilimumab combined with anti-PD-1 therapy. Cancer. 2020;126(1):86-97.

47. Ma Y, Wang Q, Dong Q, Zhan L, Zhang J. How to differentiate pseudoprogression from true progression in cancer patients treated with immunotherapy. Am J Cancer Res. 2019;9(8):1546-53.

48.• Lee JH, Long GV, Menzies AM, Lo S, Guminski A, Whitbourne $\mathrm{K}$, et al. Association between circulating tumor DNA and pseudoprogression in patients with metastatic melanoma treated with anti-programmed cell death 1 antibodies. JAMA Oncol [Internet]. American Medical Association; 2018 [cited 2021 May 7]. p. 717-21. Available from: https://jamanetwork.com/. This study demonstrated that a favourable ctDNA profile was able to distinguish true progression from pseudo-progression on immunotherapy with high sensitivity and specificity, suggesting that ctDNA may help guide response assessments.

49.• Lee R, Rothwell DG, Chow S, Shaw HM, Turajlic S, Smith N, et al. CAcTUS: A parallel arm, biomarker driven, phase II feasibility trial to determine the role of circulating tumor DNA in guiding a switch between targeted therapy and immune therapy in patients with advanced cutaneous melanoma. J Clin Oncol. 2021;39(15_suppl):TPS9587-TPS9587. Describes an ongoing Phase II clinical trial in patients with advanced cutaneous melanoma, using ctDNA to determine when patients are responding to targeted therapy in order to switch to immunotherapy; this is an important example of using ctDNA in real time to guide therapeutic decisions, which will be crucial if it is to move into the clinic.

50. Boni A, Cogdill AP, Dang P, Udayakumar D, Njauw CNJ, Sloss $\mathrm{CM}$, et al. Selective BRAFV600E inhibition enhances T-cell recognition of melanoma without affecting lymphocyte function. Cancer Res. 2010;70(13):5213-9.

51. Hu-Lieskovan S, Mok S, Homet Moreno B, Tsoi J, Robert L, Goedert L, et al. Improved antitumor activity of immunotherapy with BRAF and MEK inhibitors in BRAFV600E melanoma. Sci Transl Med. 2015;7(279):279ra41.

52. Khalili JS, Liu S, Rodriguez-Cruz TG, Whittington M, Wardell $\mathrm{S}$, Liu C, et al. Oncogenic BRAF(V600E) promotes stromal cellmediated immunosuppression via induction of interleukin-1 in melanoma. Clin Cancer Res. 2012;18(19):5329-40.

53. Luke JJ, Flaherty KT, Ribas A, Long GV. Targeted agents and immunotherapies: optimizing outcomes in melanoma. Nat Rev Clin Oncol. 2017;14(8):463-82.

54. First report of efficacy and safety from the phase II study SECOMBIT (SEquential COMBo Immuno and Targeted therapy study) I OncologyPRO.

55. Immunotherapy with ipilimumab and nivolumab preceded or not by a targeted therapy with encorafenib and binimetinib - Full Text View - ClinicalTrials.gov.

56. Marsavela G, Johansson PA, Pereira MR, McEvoy AC, Reid $\mathrm{AL}$, Robinson C, et al. The prognostic impact of circulating tumour dna in melanoma patients treated with systemic therapies-beyond braf mutant detection. Cancers (Basel). 2020;12(12):1-13.

57. Marczynski GT, Laus AC, dos Reis MB, Reis RM, Vazquez V de L. Circulating tumor DNA (ctDNA) detection is associated with shorter progression-free survival in advanced melanoma patients. Sci Rep. 2020;10(1):18682.
58.• Lee JH, Menzies AM, Carlino MS, McEvoy AC, Sandhu S, Weppler AM, et al. Longitudinal monitoring of ctDNA in patients with melanoma and brain metastases treated with immune checkpoint inhibitors. Clin Cancer Res. 2020;26(15):4064-71. This study demonstrated that ctDNA was less successful in detecting or monitoring intra-cranial disease activity compared to extra-cranial disease, highlighting a limitation of using plasma ctDNA in patients with CNS spread.

59. Momtaz P, Pentsova E, Abdel-Wahab O, Diamond E, Hyman D, Merghoub T, et al. Quantification of tumor-derived cell free DNA(cfDNA) by digital PCR (DigPCR) in cerebrospinal fluid of patients with BRAFV600 mutated malignancies. Oncotarget [Internet]. 2016 Nov 16 [cited 2021 Apr 9];7(51):85430-6. Available from: http://www.impactjournals.com/oncotarget/ index.php?journal $=$ oncotarget $\&$ page $=$ article $\& o p=$ download $\&$ path $\% 5 \mathrm{~B} \% 5 \mathrm{D}=13397 \&$ path $\% 5 \mathrm{~B} \% 5 \mathrm{D}=42512$.

60. Moya I, Villatoro S, Mayo C, Balada A, Viteri S, GonzálezCao M, et al. P05.09 Detection of clinically relevant mutations in the cerebrospinal fluid of patients with central nervous system metastases. Neuro Oncol [Internet]. 2018 Sep 19 [cited 2021 Jun 7];20(suppl_3):iii304. Available from:/pmc/articles/ PMC6144434/?report=abstract

61. De Mattos-Arruda L, Mayor R, Ng CKY, Weigelt B, MartínezRicarte F, Torrejon D, et al. Cerebrospinal fluid-derived circulating tumour DNA better represents the genomic alterations of brain tumours than plasma. Nat Commun [Internet]. 2015 Nov 10 [cited 2021 Apr 8];6(1):1-6. Available from: www.nature. com/naturecommunications.

62. Melms J, Ho K, Thummalapalli R, Tyler J, Brinker T, Singh V, et al. Implementation of cell-free tumor DNA sequencing from the cerebrospinal fluid to guide treatment in a patient with primary leptomeningeal melanoma: a case report. Mol Clin Oncol [Internet]. 2018 May 9 [cited 2021 Jun 7];9(1). Available from: https://pubmed.ncbi.nlm.nih.gov/29977540/.

63. Li Y, Pan W, Connolly ID, Reddy S, Nagpal S, Quake S, et al. Tumor DNA in cerebral spinal fluid reflects clinical course in a patient with melanoma leptomeningeal brain metastases. J Neurooncol [Internet]. 2016 May 1 [cited 2021 Jun 7];128(1):93-100. Available from: https://pubmed.ncbi.nlm.nih.gov/26961773/.

64. Ballester LY, Glitza Oliva IC, Douse DY, Chen MM, Lan C, Haydu LE, et al. Evaluating circulating tumor DNA from the cerebrospinal fluid of patients with melanoma and leptomeningeal disease. J Neuropathol Exp Neurol [Internet]. 2018 Jul 1 [cited 2021 Jun 7];77(7):628-35. Available from: https://pubmed.ncbi. nlm.nih.gov/29873738/.

65. Ulz P, Thallinger GG, Auer M, Graf R, Kashofer K, Jahn SW, et al. Inferring expressed genes by whole-genome sequencing of plasma DNA. Nat Genet. 2016;48(10):1273-8.

66. Wan JCM, Heider K, Gale D, Murphy S, Fisher E, Mouliere F, et al. ctDNA monitoring using patient-specific sequencing and integration of variant reads. Sci Transl Med. 2020;12(548).

67. McDonald BR, Contente-Cuomo T, Sammut S-J, Odenheimer-Bergman A, Ernst B, Perdigones N, et al. Personalized circulating tumor DNA analysis to detect residual disease after neoadjuvant therapy in breast cancer. Sci Transl Med. 2019;11(504):eaax7392.

68. Luo H, Wei W, Ye Z, Zheng J, Xu R hua. Liquid biopsy of methylation biomarkers in cell-free DNA. Trends Mol Med. Elsevier Ltd. 2021;27:482-500.

69. Miell JP, Taylor AM, Zini M, Maheshwari HG, Ross RJ, Valcavi R. Effects of hypothyroidism and hyperthyroidism on insulinlike growth factors (IGFs) and growth hormone- and IGF-binding proteins. J Clin Endocrinol Metab. 1993;76(4):950-5. 
70. Liu J, Chen X, Wang J, Zhou S, Wang CL, Ye MZ, et al. Biological background of the genomic variations of cf-DNA in healthy individuals. Ann Oncol. 2019;30(3):464-70.

71. Razavi P, Li BT, Brown DN, Jung B, Hubbell E, Shen R, et al. High-intensity sequencing reveals the sources of plasma circulating cell-free DNA variants. Nat Med. 2019;25(12):1928-37.

72. Smerage JB, Barlow WE, Hortobagyi GN, Winer EP, LeylandJones B, Srkalovic G, et al. Circulating tumor cells and response to chemotherapy in metastatic breast cancer: SWOG S0500. J Clin Oncol. 2014;32(31):3483-9.

73. Rustin GJS, van der Burg MEL, Griffin CL, Guthrie D, Lamont A, Jayson GC, et al. Early versus delayed treatment of relapsed ovarian cancer (MRC OV05/EORTC 55955): a randomised trial. Lancet (London, England). 2010;376(9747):1155-63.

Publisher's Note Springer Nature remains neutral with regard to jurisdictional claims in published maps and institutional affiliations. 